\title{
Article \\ Pilot Study on Poultry Meat from Antibiotic Free and Conventional Farms: Can Metagenomics Detect Any Difference?
}

\author{
Alessandra De Cesare ${ }^{1, *}$, Chiara Oliveri ${ }^{2}$, Alex Lucchi $^{2}$, Federica Savini ${ }^{1}$, Gerardo Manfreda ${ }^{2}$ \\ and Claudia Sala ${ }^{3}$ (D) \\ 1 Department of Veterinary Medical Sciences, University of Bologna, Ozzano dell'Emilia, \\ 40064 Bologna, Italy; federica.savini3@unibo.it \\ 2 Department of Agricultural and Food Sciences, University of Bologna, Ozzano dell'Emilia, \\ 40064 Bologna, Italy; chiara.oliveri5@unibo.it (C.O.); alex.lucchi3@unibo.it (A.L.); \\ gerardo.manfreda@unibo.it (G.M.) \\ 3 Department of Physics and Astronomy, University of Bologna, 40126 Bologna, Italy; claudia.sala3@unibo.it \\ * Correspondence: alessandra.decesare@unibo.it; Tel.: +39-0512097583
}

Citation: De Cesare, A.; Oliveri, C.; Lucchi, A.; Savini, F.; Manfreda, G.; Sala, C. Pilot Study on Poultry Meat from Antibiotic Free and Conventional Farms: Can Metagenomics Detect Any Difference? Foods 2022, 11, 249. https://doi.org/10.3390/ foods11030249

Academic Editor: Karl R. Matthews

Received: 16 December 2021

Accepted: 13 January 2022

Published: 18 January 2022

Publisher's Note: MDPI stays neutral with regard to jurisdictional claims in published maps and institutional affiliations.

Copyright: (c) 2022 by the authors. Licensee MDPI, Basel, Switzerland. This article is an open access article distributed under the terms and conditions of the Creative Commons Attribution (CC BY) license (https:// creativecommons.org/licenses/by/ $4.0 /)$.

\begin{abstract}
Antibiotic free farms are increasing in the poultry sector in order to address new EU regulations and consumer concerns. In this pilot study, we investigated whether the efforts of raising chickens without the use antibiotics make any difference in the microbiome of poultry meat eaten by consumers. To this aim we compared the microbiomes characterizing caeca and the corresponding carcasses of two groups of chickens reared, one reared on a conventional farm and one on an antibioticfree intensive farm. The results showed a clear separation between the taxonomic, functional and antibiotic resistant genes in the caeca of the birds reared on the conventional and antibiotic free farm. However, that separation was completely lost on carcasses belonging to the two groups. The antibiotic-free production resulted in statistically significant lower antimicrobial resistance load in the caeca in comparison to the conventional production. Moreover, the antimicrobial resistance load on carcasses was much higher than in the caeca, without any significant difference between carcasses coming from the two types of farms. All in all, the results of this research highlighted the need to reduce sources of microbial contamination and antimicrobial resistance not only at the farm level but also at the post-harvest one.
\end{abstract}

Keywords: broiler; antibiotic free farms; conventional farms; microbiome; resistome; shotgun metagenomic sequencing

\section{Introduction}

In 2019 the European Union (EU) produced an estimated 13.3 million tonnes of poultry meat, representing an increase of around $27 \%$ in comparison to 2010 [1]. Poultry meat is characterized by high-quality proteins, vitamins, and minerals important for the human diet [2]. Since the poultry rearing cycle lasts 35 to 42 days, poultry meat can be produced without the use of antimicrobials more easily than pork and beef, which have rearing cycles that last for months. Moreover, the mean values, expressed in number of defined daily doses (DDDvet)/biomass for poultry, of antimicrobial agents obtained from the technical estimates of the sales of veterinary antimicrobials in the European Union in 2016 were 0.5 for poultry versus 1.3 for pigs [3].

There are different strategies to achieve antibiotic free poultry flocks such as the implementation of effective biosafety measures and management options as well as the promotion of beneficial microbes in the gastrointestinal (GI) tract of chickens to enhance animal health and inhibit pathogen colonization. To this aim, feed can be supplemented with probiotics and prebiotics, and also blended in the same supplement (i.e., a symbiotic), to ensure diversity and stability of the GI microbial community, as well as positive 
interactions with the host's gastroenteric epithelium and immune system [4]. As an alternative, probiotics can also be supplemented in the litter and up taken by the animals [5]. Whenever a poultry disease occurs in an antibiotic free flock the animals are treated with antibiotics and the flock turns into conventional. Therefore, poultry farms can occasionally rear antibiotic free flocks along with conventional ones.

In the last decades, the microorganisms colonizing the chicken gut have been mainly investigated using classical microbiological cultural methods. However, the application of high-throughput sequencing techniques is now allowing a deeper investigation of the whole microbial composition of the chicken GI tract, including strictly anaerobic and not cultivable microorganisms. With reference to the high-throughput sequencing techniques, Durazzi et al. [6] compared taxonomic results obtained by metataxonomics (i.e., 16S rRNA gene sequencing) and metagenomics (i.e., whole shotgun metagenomic sequencing) to investigate their reliability for bacteria profiling of the chicken GI tract. The results showed that shotgun sequencing has more power to identify less abundant taxa than $16 \mathrm{~S}$ sequencing, and that the low abundant taxa have a relevant biological meaning, being able to discriminate between the experimental conditions as much as the more abundant genera detected by both sequencing strategies. Moreover, shotgun metagenomics allows for the investigation not only of the microbial population, but also of the composition in functional genes, including antibiotic resistant ones [7-9].

The increasing incidence of antibiotic resistance is considered one of the major threats to global public health $[10,11]$. In the European Union, antimicrobial resistance is responsible for 33,000 human deaths per year [12]. The use of antibiotics in animal food productions has been considered the primary cause of shedding of antibiotic resistant pathogenic and commensal bacteria [13], which can then be transferred to humans via several pathways, ref. [14] including the food-chains [15,16]. Tang et al. [17] demonstrated that reducing the level of antibiotic use in animal productions can be effective to fight antibiotic resistance in both animals and humans. To this aim, many initiatives and strategies have been put in place by both policy makers and producers and as a possible consequence, in 2017, for the first time since 2011, the antimicrobial consumption in food producing animals was lower than in humans (i.e., $108.3 \mathrm{mg} / \mathrm{kg}$ vs. $130 \mathrm{mg} / \mathrm{kg}$ ) [3].

To limit the use of antibiotics in animal rearing, Regulation (EC) No. 1831/2003 [18] banned the marketing and use of antibiotics as growth promoters in feed in the European Union since January 2006. Furthermore, starting from January 2022, the new Regulations EU 2019/6 [19] on Veterinary Medicinal Products and 2019/4 [20] on Medicated Feed will enter in force as further steps in the EU strategy to fight antimicrobial resistance. Other mitigation measures include the prevention of bacterial diseases in the animals, the use of specific antimicrobials for food producing animals but not for humans, the improvement of biosecurity protocols at farm level, the wide use of vaccination programs, and the use of nutrients and additives in feed promoting the immune system and supporting beneficial microbes [21].

In the pilot study described in this paper we investigated whether the efforts of raising chickens without the use of antibiotics made any difference in the microbiome of poultry that consumers eat. To this end, we compared the microbiomes characterizing caeca and the corresponding carcasses of two groups of chickens reared on different farms-one conventional and one antibiotic-free. Moreover, with the view of planning future studies, we investigated whether checking the correlation between the microbiome and resistome in the caeca and the carcass of the same animal provides more insight than the same analysis performed at flock level.

\section{Materials and Methods}

\subsection{Samples and Sampling Plan}

A total of 30 slaughtered broilers were randomly sampled at the slaughterhouse within the first group of animals processed at the start of the working day, when the slaughterhouse was still clean and disinfected to avoid bias due to cross-contamination. Among the selected 
broilers, 15 belonged to a poultry flock reared in a conventional farm and treated with Amoxicillin (20-30 mg/ $\mathrm{kg}$ of live weight in drinking water for three to five days) at days eight and 29 and with sulfadimethoxine/trimethoprim $(100 \mathrm{mg} / 20 \mathrm{mg}$ in 1-2 L of drinking water once a day for five days) at day 24 . The remaining 15 belonged to a poultry flock reared in a conventional farm but never treated with antibiotics. All tested animals were female, ROSS, fed with no medicated feed and slaughtered at 47 (antibiotic free) and 48 (conventional) days. For each sampled animal, both the gastrointestinal tract and the carcass were collected. The GI tract was sampled during slaughtering, at the evisceration step, and immediately stored in a sterile plastic bag kept at $4{ }^{\circ} \mathrm{C}$ in a refrigerated box. Moreover, a sterile plastic flag was attached to the hook transporting the carcass from which the GI tract was sampled in order to pick up that specific carcass at the end of the refrigeration tunnel. After sampling, each carcass was stored in a sterile plastic bag kept at $4{ }^{\circ} \mathrm{C}$ in a second refrigerated box, different from that containing the GI tracts. All samples were transported to the laboratory within two hours and immediately processed.

Each GI tract was dissected and a small portion (i.e., 0.5 to $2 \mathrm{~g}$ ) of caeca content was collected, transferred into a $2 \mathrm{~mL}$ sterile plastic tube, and flash frozen in liquid nitrogen before storage at $-80^{\circ} \mathrm{C}$ until DNA extraction. A total of $10 \mathrm{~g}$ of neck and breast skin were collected from each carcass, diluted in $90 \mathrm{~mL}$ of sterile physiological solution $(\mathrm{NaCl} 0.90 \%)$, homogenized in the stomacher (MAYO HG 400V, Italy) at normal speed for 1 min and centrifuged at $4{ }^{\circ} \mathrm{C}$ for $20 \mathrm{~min}$ at $9980 \times \mathrm{g}$. The obtained pellet containing the concentrated cells was stored at $-80^{\circ} \mathrm{C}$ until DNA extraction.

\subsection{DNA Extraction and Sequencing}

The DNA was extracted using a bead-beating procedure followed by the QIAmp ${ }^{\circledR}$ DNA Stool Mini Kit (Qiagen, Milan, Italy) for samples of caeca content [22], and by PowerFood ${ }^{\circledR}$ Microbial DNA Isolation Kit (MO BIO-Qiagen) for the pellet obtained from each carcass [23]. The DNA extracted from each sample was quantified using a BioSpectrometer ${ }^{\circledR}$ (Eppendorf, Milan, Italy) to assess DNA yield, in terms of quantity and quality. Total DNA from caeca and carcass samples was fragmented and tagged with sequencing indexes and adapters using the Nextera XT DNA Library Preparation Kit (Illumina, San Diego, CA, USA). Shotgun metagenomic sequencing was performed using the NextSeq500 (Illumina) $2 \times 150 \mathrm{bp}$ in paired-end mode. One caeca sample was removed later in the process for technical reasons (linked to sequencing yield) resulting in a total of 59 metagenomes: 29 from broilers caeca and 30 from carcasses.

\subsection{Bioinformatic and Biostatistic Analysis}

Filtering and trimming of raw reads were performed using MG-RAST https:/ / www. mg-rast.org (accessed on 14 December 2021) [24] bioinformatics pipelines. In the taxonomic analysis, only taxa from the bacterial domain were considered. Moreover, taxa present in less than 4 samples or represented by less than four reads were discarded. Analogously, in the functional analysis, functional genes present in less than four samples or represented by less than 4 reads were discarded.

The statistical analysis of both the taxonomic and functional gene composition was performed using R 3.6.3 and the libraries phyloseq 1.30.0 [25] and DESeq2 1.26.0 [26]. Relative abundances displayed in the bar plots were computed normalizing to sum to 1 the read counts obtained from MG-RAST. Then, in the bar plots only the first (at most) 20 taxa/functional elements with relative abundance greater than $1 \%$ were shown.

Before proceeding with the statistical analysis, read counts were normalized with DESeq2 to take into account the different sizes of the samples. In this step, size factors were estimated using the function estimateSizeFactors of DESeq2 with the "poscount" option, as suggested when dealing with sparse data. Then, DESeq2 was used to assess whether the taxa/function abundances differed between groups. Specifically, the Wald test was used to determine the statistical significance and the Log Fold Changes were shrunk using the apeglm method [27]. Finally, p-values were adjusted for multiple testing using the 
Benjamini-Hocheberg procedure [28]. A threshold of 0.05 was used in all analyses to assess their statistical significance.

Alpha diversity was estimated using the InvSimpson/Shannon/Chao1 index, and differences in alpha diversity between groups were evaluated fitting a linear regression model and using the Student's $t$-test to assess whether the linear relationship between alpha diversity and the grouping was negligible. Beta diversity was estimated starting from the read counts normalized with DESeq2 and computing the Bray-Curtis dissimilarity [29] among samples. Principal Coordinate Analysis (PCOA) was used to visualize the results. After applying the rlog transformation [26] to DESeq2 normalized counts, Principal Component Analysis (PCA) was performed using the function prcomp of the library stats 3.6.3 in $\mathrm{R}$ and the correlation between samples was computed using Kendall's coefficient.

The resistome of each sample was predicted using the Resistance Gene Identifier (RGI) [30]. Fastq reads were aligned using the bowtie2 algorithm [31] to the 'canonical' curated CARD reference sequences [30], as well as to the in silico predicted allelic variants available in CARD's Resistomes \& Variants data set [30], as suggested in the resistance gene identifier (RGI). The alignments were obtained at the allele level and were filtered so that only entries with $>95 \%$ identity to the CARD reference sequences and with more than 50 base pairs of reference allele covered by reads were kept. RGI mapping counts were adjusted for differences in both gene lengths and bacterial sequence abundances by computing fragments per kilobase reference per million bacterial fragments (FPKM). Results at the AMR gene family and drug class level were obtained by aggregating the counts at the allele level. The beta-diversity of the samples based on the resistome was obtained by computing the PCoA. To this aim, the counts were normalized with DESeq2 as previously described, and the Bray-Curtis distances between all samples were calculated using the R packages vegan 2.5.7 [32] and phyloseq 1.28.0 [25]. The PCoA was computed separately for caeca and carcass samples, and the effect of the origin of the sample on the sample dissimilarities were determined using permutational multivariate analysis of variance using distance matrices (the 'adonis2' function in the vegan v2.5.7 package). Finally, conventional and antibiotic-free AMR gene families were compared using the same DESeq2 pipeline previously described for the taxonomic and functional analysis.

\section{Results}

Metagenomic sequencing yielded an average of $5 \mathrm{Gbp} /$ sample. The 59 metagenomes sequenced are publicly available from MG RAST at https:/ / www.mg-rast.org/mgmain. html?mgpage=project\&project=mgp89213 (accessed on 14 December 2021) and described in Table S1.

\subsection{Taxonomic and Functional Gene Composition of Caeca}

The beta diversity index (generated using the Bray-Curtis distance metric) calculated at the genus level highlighted a clear dissimilarity in the community composition in the caeca sampled in the two groups of broilers tested (adonis2 $p$-value $<0.00001$ ) (Figure 1).

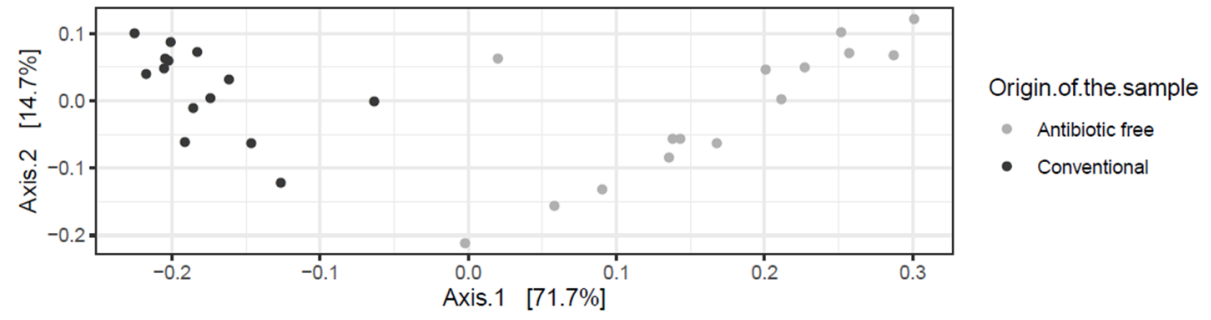

Figure 1. Bray-Curtis dissimilarity plots showing the genera detected in the caeca of birds from the conventional and antibiotic free farm.

Sixteen of the top 20 most abundant genera identified within the caeca were shared between the two tested groups (Figure 2). Moreover, Alkaliphilus, Desulfibacterium, Bacillus 
and Ethanoligenens were only detected in the caeca of birds from the conventional farm (Figure 2a), while Coprococcus, Escherichia, Parabacteroides and Provotella were detected in the caeca of birds from the antibiotic-free farm (Figure $2 b$ ).

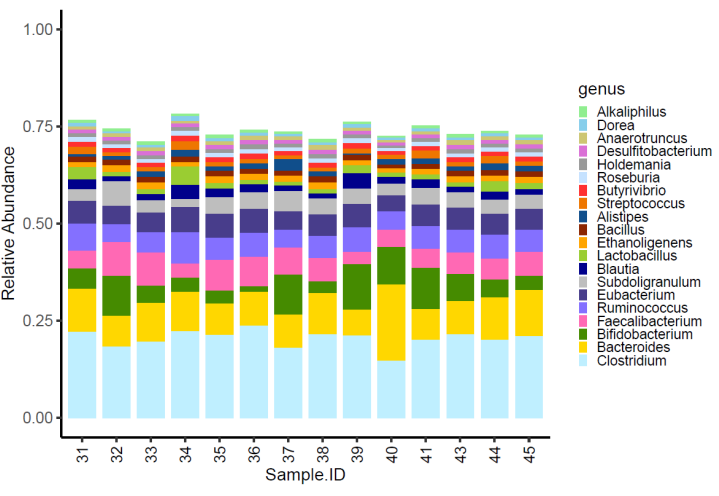

(a)

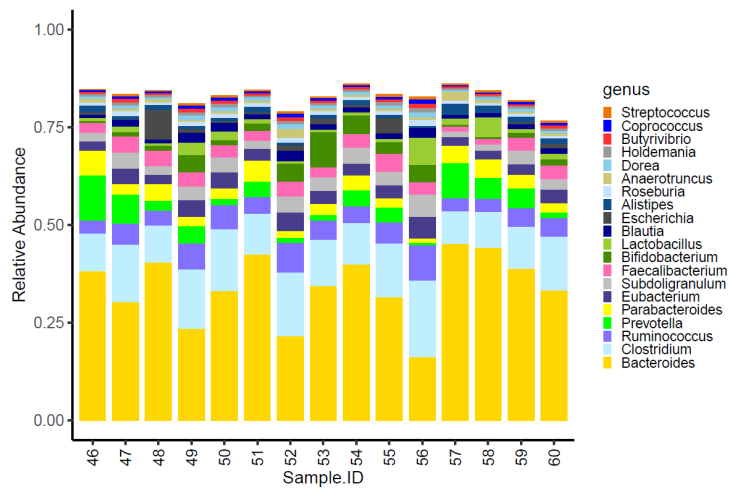

(b)

Figure 2. Top 20 genera characterizing the caeca of the birds reared in the conventional (a) and antibiotic free (b) farm. Sample 42 is not included among samples in panel a because it was not processed for technical reasons.

The normalized mean counts of Alkaliphilus, Bacillus, Desulfitobacterium, Ethanoligenens and Streptococcus were significantly higher in the caeca of birds reared on the conventional farm, while those of Alistipes, Anaerotruncus, Bacteroides, Coprococcus, Dorea, Escherichia, Holdemania, Lactobacillus, Parabacteroides, Prevotella, Roseburia, Ruminococcus and Subdoligranulum were higher in the caeca of birds reared on the antibiotic-free farm (Table S2). In terms of alpha diversity, representing richness and diversity of the genera within the caeca, the InvSimpson, Shannon and Chao1 indexes were significantly higher in the caeca of birds from the group of animals reared in the conventional in comparison to the antibiotic free farm (Table S3 and Figure 3).

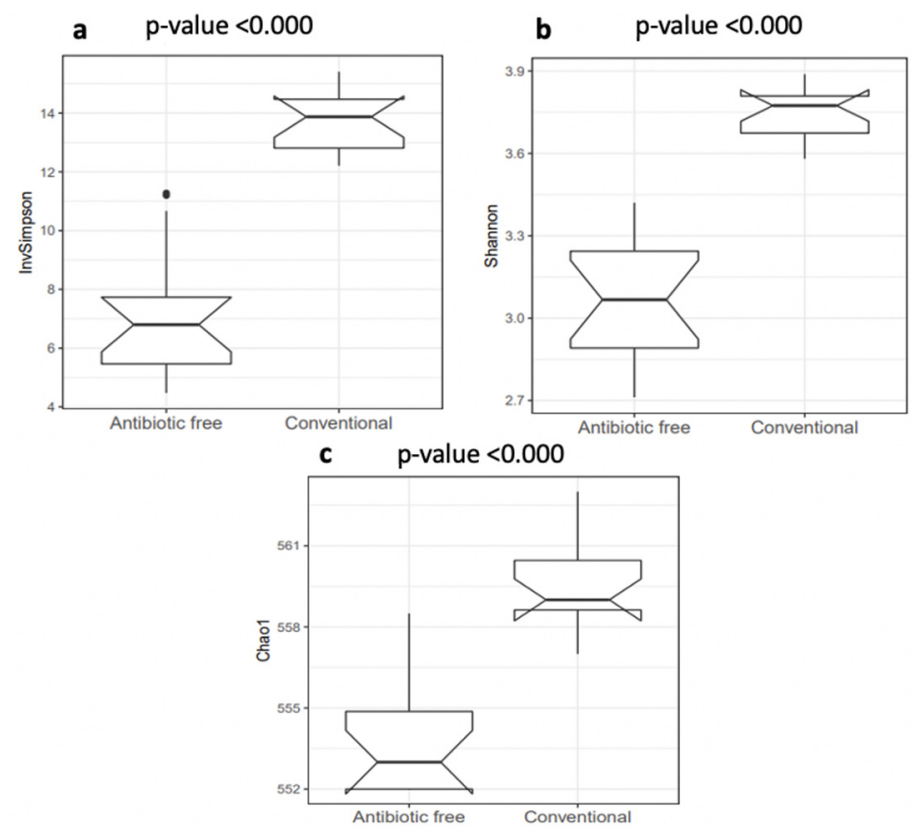

Figure 3. Box plots of the alpha diversity indexes calculated at genus level: (a) InvSimpson index; (b) Shannon index; (c) Chao1 index. $p$ values $<0.001$ were considered significantly different.

The beta diversity index calculated for the functional genes confirmed a clear association of the caeca with their farm of origin (adonis2 $p$-value $<0.00001$ ) (Figure 4). 


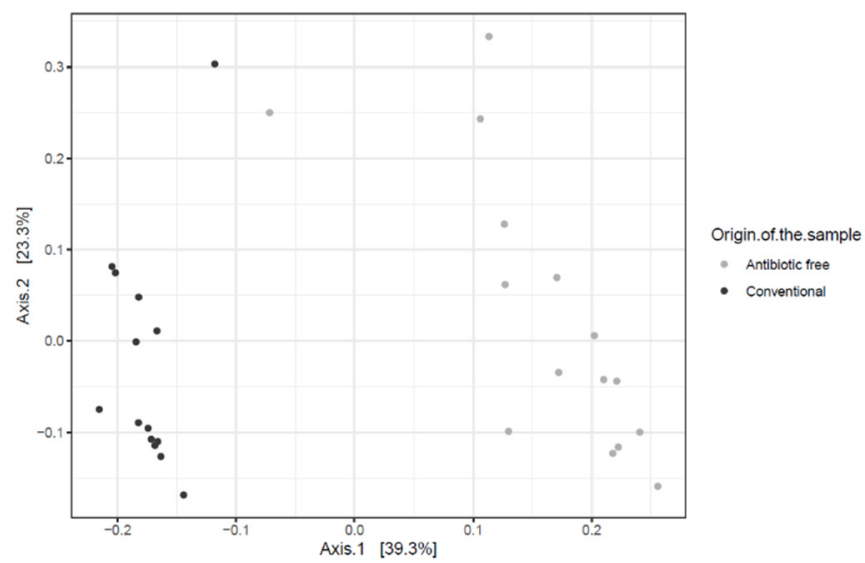

Figure 4. Bray-Curtis dissimilarity plots showing the functional genes detected in caeca of birds reared in the conventional and antibiotic free farm.

Fourteen of the top 20 most abundant functional genes identified within the caeca of birds were shared between the two tested groups (Figure 5). Moreover, functional genes type I restriction-modification system-restriction subunit $\mathrm{R}$ (EC 3.1.21.3), site-specific recombinase, DNA topoisomerase III (EC 5.99.1.2), ferrous iron transport protein B, copper-translocating P-type ATPase (EC 3.6.3.4) and DNA gyrase subunit A (EC 5.99.1.3) were listed among the most abundant functional genes in the caeca of birds from the conventional farm, while chaperone protein DnaK, glutamine synthetase type III, GlnN (EC 6.3.1.2), leucyl-tRNA synthetase (EC 6.1.1.4), valyl-tRNA synthetase (EC 6.1.1.9), clpB protein and tonB-dependent receptor were only detected in the caeca of birds from the antibiotic-free farm.
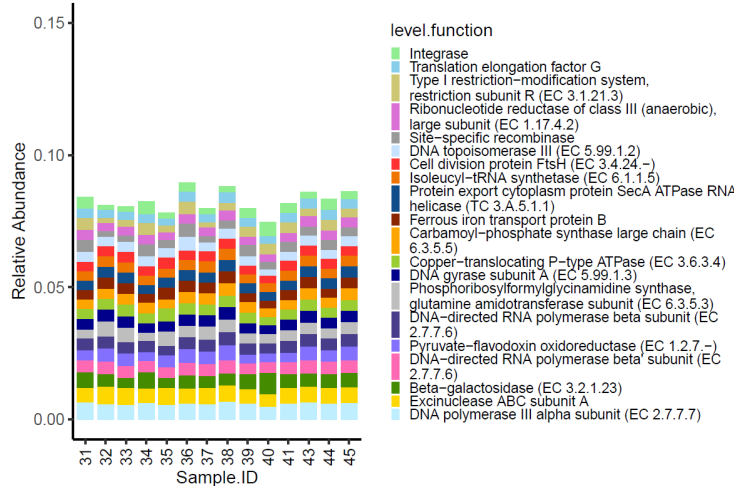

(a)

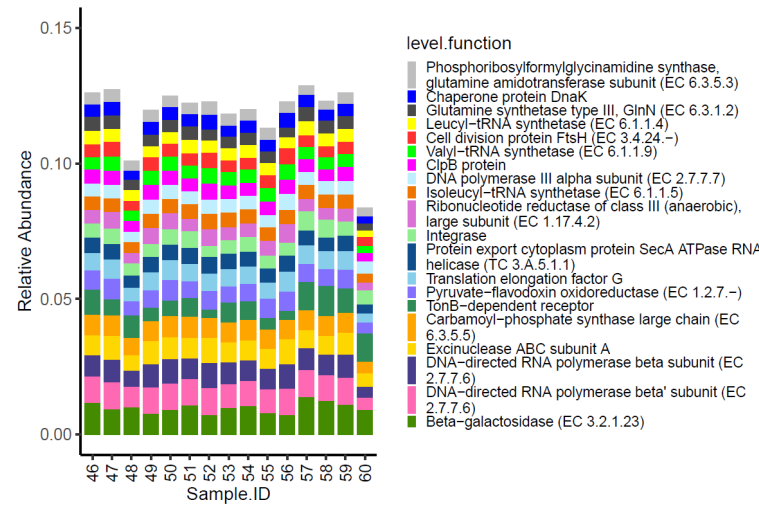

(b)

Figure 5. Top 20 functional genes characterizing the caeca of the birds from the conventional (a) and antibiotic free (b) farm. Sample 42 is not included among samples in panel a because it was not processed for technical reasons.

Besides these qualitative differences, the normalized mean values of beta-galactosidase (EC 3.2.1.23) and DNA topoisomerase III EC 5.99.1.2 (in PFGI-1-like cluster) were significantly higher in the caeca of birds from the conventional farm, while those of integrase, translation elongation factor $\mathrm{G}$, ribonucleotide reductase of class III (anaerobic)-large subunit EC 1.17.4.2, excinuclease ABC subunit A paralog were greater in the Bacteroides group, and the chaperone protein DnaK was significantly higher in the caeca of birds reared in the antibiotic free farm (Table S4).

\subsection{Taxonomic and Functional Composition of Carcasses}

The genera identified in the carcasses showed a slight clustering (adonis2 $p$-value $=0.020$ ) according to the farm of origin (Figure S1). Overall, 14 of the top 20 most abundant genera 
identified in the carcasses were shared between the two tested groups (Figure 6). Moreover, Anoxybacillus, Bacillus, Flavobacterium, Pedobacter, Geobacillus and Sphingobacterium were only detected on carcasses of birds from the conventional farm (Figure 6a), while Aeromonas, Burkholderia, Endoriftia, Prevotella, Ruminococcus and Shewanella were only detected on carcasses of birds from the antibiotic-free farm (Figure 6b).

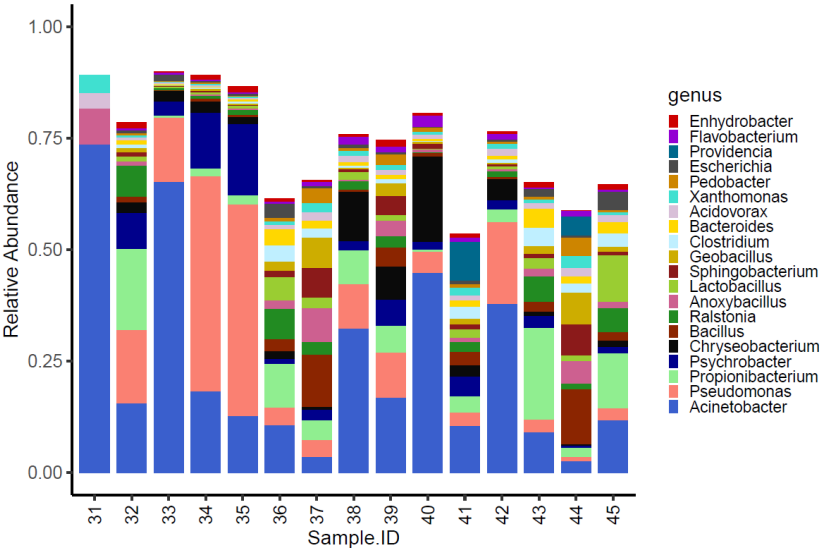

(a)

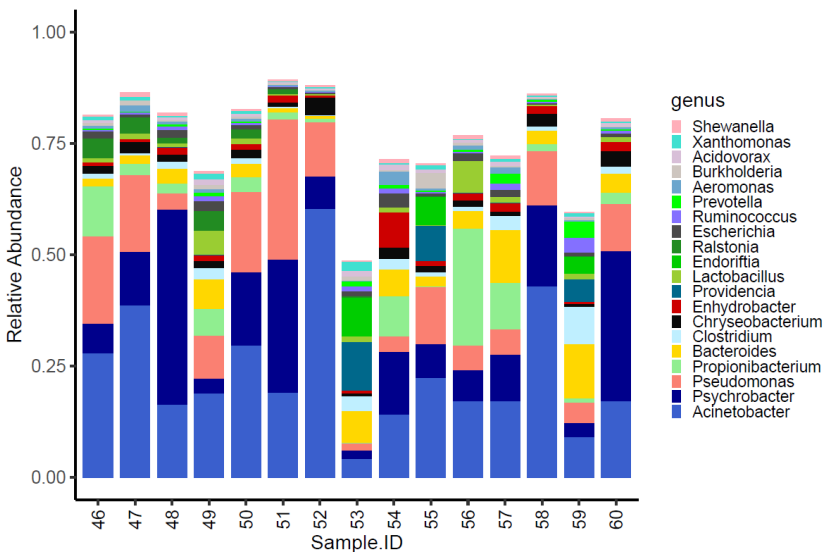

(b)

Figure 6. Top 20 genera characterizing the carcasses of the birds reared in the conventional (a) and antibiotic-free (b) farm.

The normalized mean values of Anoxybacillus, Bacillus, Geobacillus, Pedobacter, and Sphingobacterium were significantly higher on carcasses of birds reared in the conventional farm, while those of Aeromonas, Bacteroides, Prevotella and Ruminococcus were higher in the carcasses of birds reared on the antibiotic-free farm (Table S5). The alpha diversity indexes (i.e., InvSimpson, Shannon and Chao1) calculated for bacteria genera identified on carcasses from the conventional and the antibiotic-free farm did not show any significative difference (Table S6). Moreover, the beta diversity calculated for the functional genes did not group the carcasses according to the farm of origin (adonis2 $p$-value $=0.332$ ) (Figure S2).

Three of the top 20 most abundant functional genes identified on the carcasses were shared between the two tested groups (Figure 7). Moreover, the functional genes cytochrome c oxidase polypeptide II (EC 1.9.3.1) and DNA-directed RNA polymerase beta' subunit (EC 2.7.7.6) were listed among the most abundant functional genes on carcasses of birds from the conventional farm, while 2-oxoglutarate dehydrogenase E1 component (EC 1.2.4.2), RNA helicase putative and DNA topoisomerase I (EC 5.99.1.2) were most abundant only on carcasses of birds from the antibiotic-free farm.

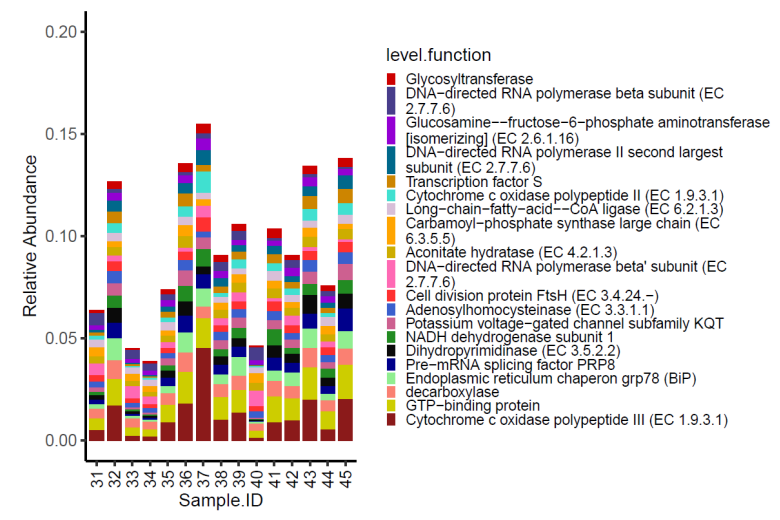

(a)

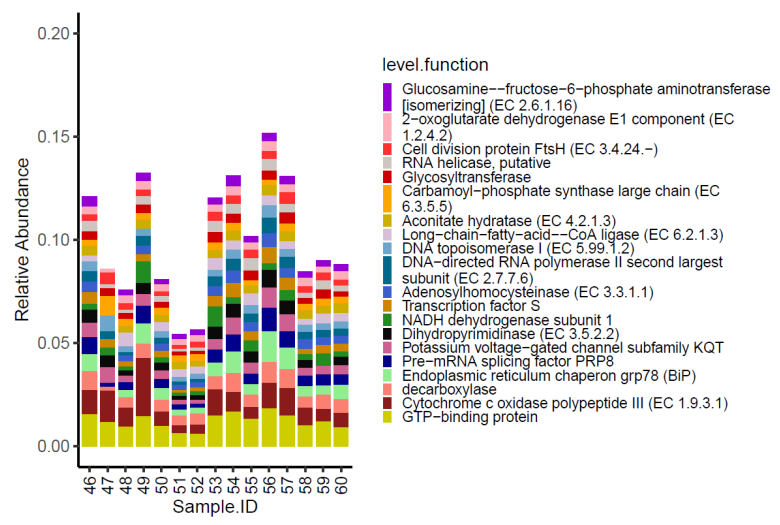

(b)

Figure 7. Top 20 functional genes characterizing the carcasses of the birds from the conventional (a) and antibiotic free (b) farm. 
As for the genera, the functional genes did not show significative different normalized mean counts between the two groups of carcasses tested $(p<0.05)$.

\subsection{Longitudinal Analysis of Caeca and Carcass Microbiomes Belonging to the Same Animal}

In Figure 8 the correlations between caeca samples and carcasses are shown. Both the dimension of the circle and the color scale represent the value of the correlation coefficient. Overall, a positive correlation was always detected between the genera colonizing the caeca and the corresponding carcass. However, such correlation was not stronger for samples collected from the same animal than for animals belonging to the same flock. As an example, bacteria genera identified in the caeca of the bird labelled as 31 in Figure 8a are well correlated to those of carcass 31 as well as to carcasses 36, 37, 43 and 45. On the contrary, the bacteria genera identified in the caeca of the bird labelled as 50 in Figure $8 \mathrm{~b}$ show a higher correlation with the carcasses 47 and 59 in comparison to the carcass of the same animal (i.e., carcass 50). A positive correlation was also identified between functional genes detected in the caeca and corresponding carcass for chickens reared in the conventional (Figure 8c) and antibiotic free (Figure 8d) farms. For the functional genes, such positive correlation was higher in comparison to that observed for the bacteria genera, thus resulting in stronger blue dots.

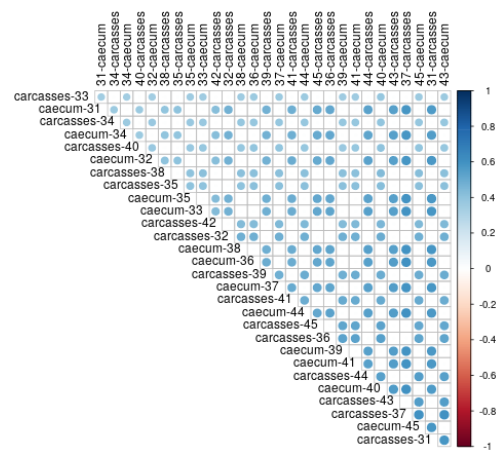

(a)

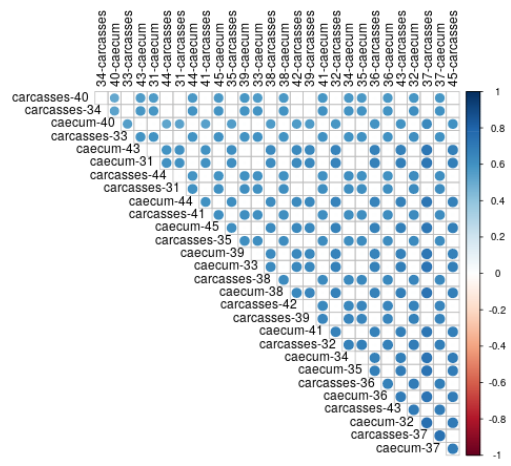

(c)

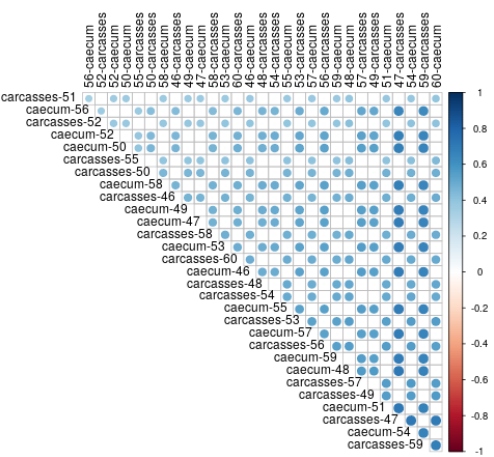

(b)

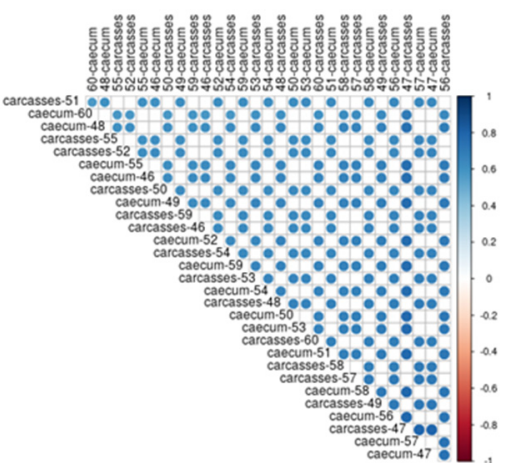

(d)

Figure 8. Kendall correlation plot of genera (a) and functional genes (c) identified in the caeca and corresponding carcasses from the conventional farm and of genera (b) and functional genes (d) identified in the caeca and corresponding carcasses from the antibiotic-free farm.

\subsection{Identification of Antibiotic Resistance Genes in the Caeca and Carcass Microbiome}

The antibiotic resistance genes (ARGs) were retrieved from functional genes identified in the caeca and in the carcasses of the tested animals and classified as such in level 2 of the biological-function ontology in the SEED category [33]. Among the ARGs with normalized mean values of abundance $>1000$ in at least one tested group, the regulatory sensor-transducer-BlaR1/MecR1 family, UDP-N-acetylmuramoylalanyl-D-glutamate-2,6diaminopimelate ligase (EC 6.3.2.13), macrolide export ATP-binding/permease protein 
MacB (EC 3.6.3.-), multi antimicrobial extrusion protein $(\mathrm{Na}(+)$ /drug antiporter)-MATE family of MDR efflux pumps, topoisomerase IV subunit B (EC 5.99.1.-) and vancomycin response regulator VanR were significantly higher in the caeca of birds reared in the conventional farm in comparison to the antibiotic-free farm (Table S7). On the contrary, the acriflavin resistance protein was significantly higher in the caeca of birds reared on the antibiotic-free farm (Table S7). As far as the carcasses are concerned, no differences were identified between normalized mean values of abundance of ARGs detected on carcasses sampled in the two tested groups. The total antimicrobial resistance (AMR) load (Figure 9) was significantly higher in the caeca of birds reared on the conventional farm in comparison to the antibiotic-free farm (Wilcoxon rank sum test $p$-value $<0.000$ ) (Figure 9). However, in both groups of caeca samples, the total AMR load was lower in comparison to the carcasses which did not show significant differences between the two tested groups (Wilcoxon rank sum test $p$-value $=0.6$ ).

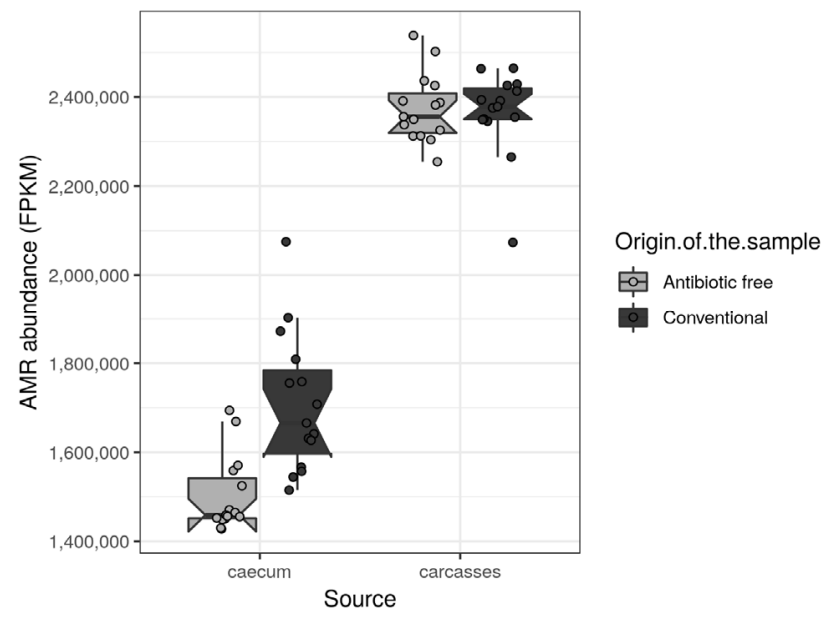

Figure 9. Box plots showing the total AMR level (FPKM) per sample, stratified by source and origin of the sample. Each sample is also represented by a dot with sideways jitter to minimize overplotting.

The drug classes identified in the broiler caeca and on carcasses are listed in Figure 10. The drug class aminoglycoside was more represented on carcasses in comparison to caeca contents and sulfonamide was identified on carcasses but at very low levels in some caeca. On the contrary, macrolide as well as resistance to other drug classes, including bicyclomicyn, lincosamide, fosfomycin, glycopeptide, pleuromutilin and nitrofuran were mostly identified in the caeca. Figure 10 shows that besides differences in the abundance of specific antibiotic resistance genes described above, in qualitative terms the overall resistome of the caeca of animals reared on the antibiotic-free farm overlaps with that of animals reared on the conventional farm, and the same is observed on carcasses. This result can be explained considering that the antibiotic free flock was reared in a farm where antibiotics have been possibly used in the previous flocks, thus supporting the persistence of ARGs in the farm environment over time.

As for bacteria genera and functional genes, the ARGs identified in the caeca also clustered separately for the conventional and antibiotic free-farms (adonis2 $p$-value $=0.00001$ ) (Figure 11), while in the carcasses this difference was lost (adonis2 $p$-value $=0.4278$ ) (Figure S3). 


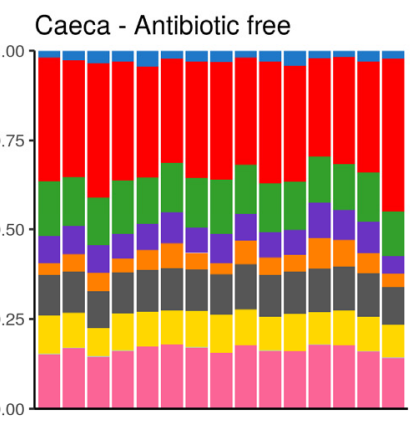

Carcasses - Antibiotic free

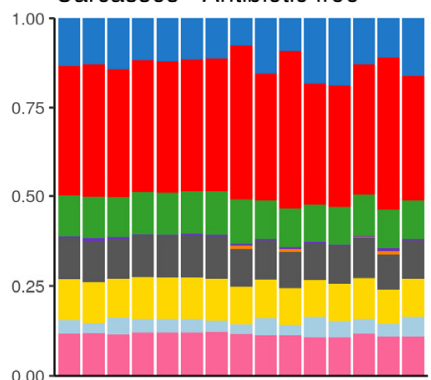

Caeca - Conventional

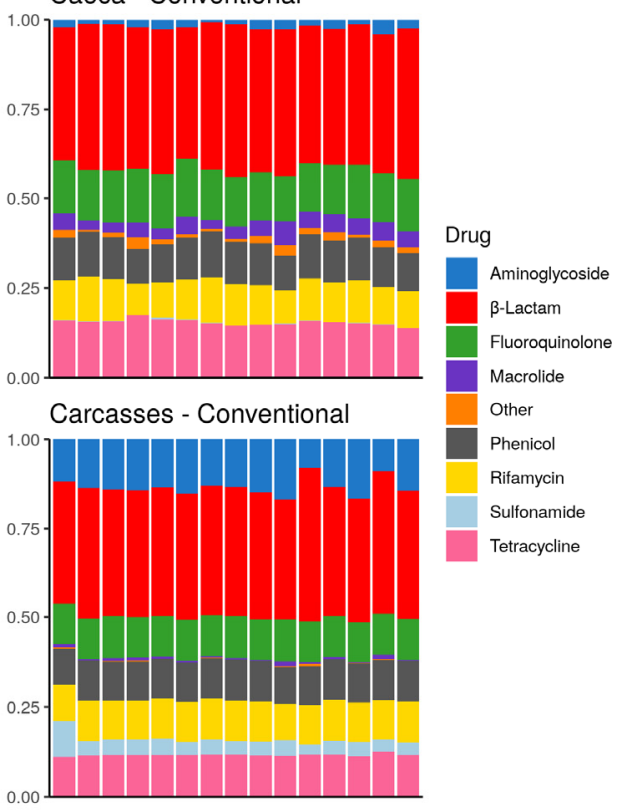

Figure 10. Stacked bar chart of antimicrobial resistance abundance (FPKM) per drug class (colors) per sample ( $\mathrm{x}$ axis). Each plot refers to one group of samples as detailed in the sub-titles.

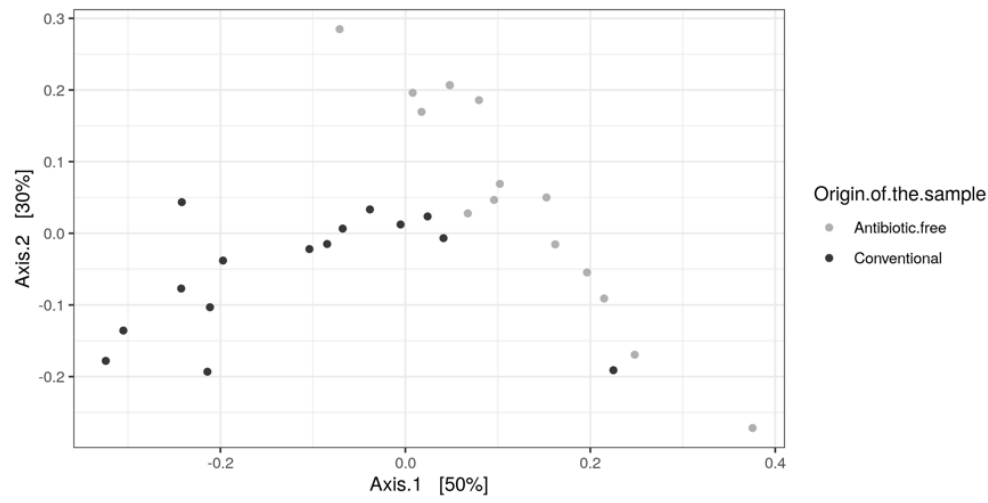

Figure 11. Beta diversity of caeca samples shown as PCoA of Bray-Curtis diversity computed based on the AMR gene family abundances normalized with DESeq2.

\section{Discussion}

Currently, conventionally raised poultry continues to dominate the EU poultry industry. However, there is an increasing consumer demand for meat obtained in antibiotic-free rearing cycles. Moreover, in January 2022, the new EU regulations of June 2019 [19] and April 2019 [20] will enter in force, further limiting the use of veterinary medical products and medicated feed in animal productions. In this pilot study, we investigated whether the efforts of raising chickens without the use antibiotics would make any difference in the microbiome of poultry meat eaten by consumers.

The results demonstrated three key findings. The first one is a clear separation between the taxonomic, functional and antibiotic resistance genes in the caeca of the birds reared on the conventional and antibiotic-free farms. This result is due to the fact that each poultry farm has an associated ecosystem due to the geographical and specific environmental conditions, to what chickens eat and drink, to the litter type, to the workers, and certainly to the medications they receive or not. That separation was completely lost on carcasses belonging to the two groups, which did not mirror whatever positive or negative impact the farm ecosystem and rearing conditions had on the chicken caeca. As for the caeca, and also for the carcass microbiomes, there are many contributing factors besides the possible cross 
contamination during the evisceration. Indeed, the ecosystems interacting with the animals during transport and then during each slaughtering step, including the final refrigeration tunnel, all contribute to the final carcass microbiomes.

The second key finding is that the antibiotic free production resulted in statistically significant lower antimicrobial resistance loads in the caeca of chickens in comparison to the conventional production, thus confirming that besides external sources of ARGs, when antimicrobials are not administered to the animals in the caeca of that flock there is a lower antimicrobial resistance load. In relation to the short- and long-term effects of the use of antimicrobials on antimicrobial resistance, Mughini-Gras et al. 2021 [34] showed that the antimicrobial use at flock level is more relevant for antimicrobial resistance in Escherichia coli than the historical use of antimicrobials at the farm level. Overall, these observations demonstrate that the effort of reducing antimicrobial use by means of rearing antibiotic free flocks should be associated with a better understanding of the antimicrobial resistance persistence in the farm environment in the absence of direct antimicrobial use. Further insights into the antimicrobial resistance persistence in the farm environment might help us to understand why, for instance, the relative abundance of acriflavine resistance protein genes was higher in the caeca of antibiotic-free animals compared to conventional ones.

The last main finding is that the antimicrobial resistance load on carcasses was much higher than in the caeca, without any significative difference between carcasses coming from the two types of farming. As described above, this result demonstrated that all postharvest steps, including transport and slaughtering, but also the loading and unloading of the animals contributes not only to the microbiome colonizing the final carcass reaching the consumers, but also to its antimicrobial resistance load. Therefore, although the most important antimicrobial resistance risk factors and possible mitigation measures are still under investigation at the farm level [35], the implementation of past and future EU regulations aimed at reducing antimicrobial use for food production animals has been ensuring a significant reduction of antimicrobial resistance load at the farm level. Therefore, the same effort made for the identification of relevant sources of pathogen and spoilage microorganisms and their ARGs should be now devoted to the post-harvest steps [36]. Little currently available data demonstrate that both transport trucks and cages can contaminate the birds with bacteria and ARGs [37] and contribute to the cross contamination between the slaughterhouse and the farms [38]. Moreover, when the animals reach the slaughtering line, scalding, defeathering and evisceration can spread both microorganisms and ARGs from the animal to the environment, although some tentative steps toward reducing these cross contaminations using innovative technologies are in place [39]. Additional sources of both microorganisms and ARGs are workers, equipment, air, process water and wastewater from slaughtering [40]. All these sources together contribute to the carcass microbiome, and our results showed that at the end of the refrigeration tunnel the microbiome of carcasses from animals reared in the conventional farm overlaps with that of carcasses from birds reared in an antibiotic-free cycle. Our results are consistent with those of Li et al., 2020 [41] who investigated chicken breast microbiomes at the retail level, accounting also for the effect of the processing environment and packaging conditions. Their results confirmed that the microbiome of the chicken breast is affected by packaging in air versus under vacuum and by the processing plant where the chicken breast is processed. On the contrary, both the use of antimicrobials at the farm level as well as seasonality affected neither the composition nor the diversity of chicken breast microbiomes in terms of both alpha and beta diversities.

The alpha diversity calculated in this study at genus level using the indicators of richness (Chao1), evenness (InvSimpson) and diversity (Shannon) within the caeca samples showed values significantly higher in the caeca of birds reared on the conventional farm in comparison to the antibiotic-free farm. The bacteria biodiversity within the GI tract is considered an indicator of good health, and it was expected to be higher in the caeca of chickens not treated with Amoxicillin and Sulfadimethoxine/Trimethoprim. However, these an- 
tibiotics are only partially absorbed in the gut [42,43], and this might explain why their administration did not reduce the overall bacteria richness in the caeca. Moreover, the intestinal microbiota biodiversity is the result of different factors, as management protocols applied on the farm, animal characteristics and administered diets [44] which were possibly different in the conventional and antibiotic free farm investigated in this research. Among the most represented genera detected in the caeca, Alkaliphilus, Desulfibacterium, Bacillus and Ethanoligenens were identified as signature genera in the birds from the conventional farm, while butyrate-producing microorganisms such as Coprococcus, Roseburia and Subdoligranulum were identified in the caeca of birds reared on the antibiotic-free farm. This result highlighted that besides the higher bacteria biodiversity identified in the caeca of birds from the conventional farm, the signature genera colonizing the caeca of the birds reared on the antibiotic-free farm belonged to microbial groups supporting animal health. Indeed, butyrate fights against pathogen colonization in poultry [45] and is involved in several intestinal functions, being an energy source stimulating epithelial cell proliferation and differentiation, other than exerting an antimicrobial effect by promoting the production of peptides and stimulating the production of tight junction proteins [46].

In this pilot study we investigated for the first time the microbiome of the caeca of a bird and that of the corresponding carcass. The results showed that the caeca and carcasses of the same flock positively correlate one with the other. However, the correlation between the microbiome of the caeca and the carcass of the same bird was not stronger than that with other caeca and carcasses of the same flock. Therefore, the target analysis of caeca and carcass of the same animal does not provide any added value in comparison to the microbiome analysis at flock level. It is also clear from Figure 8 that the correlation between the functional genes was higher than for bacteria genera, possibly because the same functional gene can be shared between different bacteria genera.

Besides the qualitative and quantitative differences in the most represented functional genes identified in the caeca and on carcasses from the animals reared in the conventional and antibiotic free farm, the most relevant result concerns the antibiotic resistant genes and the total antimicrobial resistance load. In relation to the ARGs, the multi antimicrobial extrusion protein $(\mathrm{Na}(+)$ /drug antiporter)-MATE family of MDR efflux pumps was significantly higher in the caeca of birds reared on the conventional farm in comparison to the antibiotic-free farm, along with few other ARGs. The MATE gene family is widely distributed in both Gram-positive and Gram-negative bacteria and contributes to the intrinsic, acquired, and phenotypic resistance of bacterial pathogens [47]. Moreover, it can confer resistance to a specific class of antibiotics or to many drugs, thus conferring a multi-drug resistance (MDR) phenotype to bacteria [48]. In contrast, the abundance of genes encoding the acriflavin resistance protein was significantly higher in the caeca of birds reared in the antibiotic free farm. The acriflavine resistance protein is among the multidrug resistance efflux transporter proteins that belongs to the resistance modulation division superfamily (RND), conferring broad spectrum resistance to Gram-negative bacteria [49].

For both caeca and carcasses, the overall antimicrobial resistance abundance per drug class did not show significative difference between the birds collected in the two tested farms, while specific differences were observed between drug classes associated to caeca and carcasses. The drug classes identified in both caeca and carcasses largely overlap with those identified by Munk [50] in the fecal resistome investigated in European poultry farms, including Italian farms. In both studies, aminoglycoside, $\beta$-lactam, tetracycline and macrolide are widely represented, although we identified a larger proportion of $\beta$-lactam as well as rifamycin not reported by Munk et al. Our results confirmed what was observed by Li et al., 2020 [41] in relation to the absence of difference between the resistome associated to chicken breast from birds reared in conventional and antibiotic-free farms. On the contrary, the results on the antimicrobial resistance load are the opposite because we calculated a higher antimicrobial resistance load on carcasses, while Li et al. discovered a low risk of ARG accumulation on chicken breast. This result is possibly because in the US, poultry carcasses can be disinfected using chlorinated water or organic acids, while in the European 
Union the use of substances intended to remove microbial surface contamination is only permitted after a full risk analysis taking into account the results of a risk assessment based on the available scientific evidence [51].

Genes coding for resistance to vancomycin were identified among the ARGs with normalized mean values of abundance $>1000$ in at least one tested group. In accordance with other authors [40,52], vancomycin resistance genes can be identified in poultry flocks, although avoparcin has been banned by the EU since 1997. The relative abundance we estimated for the vancomycin resistance genes constitutes a body of evidence of their persistence, while Savin et al. [40] reported a declining trend.

Overall, the results of this pilot study and the scientific literature demonstrate that each intervention in whatever processing step that the chicken and poultry meat is at, as with other food productions, is affected by the existing microbiome and resistome shifting and changing from farm to fork. Therefore, building robust, comparable, and representative databases of animal-, farm-, food- and production environment-associated microbiomes and resistome from farm to fork, as it is done for individual foodborne isolates and indicator microorganisms [53], would certainly help to predict the effect of control strategies to reduce food contamination by foodborne pathogens as well as ARGs in a systemic way.

\section{Conclusions}

All in all, the results of this research indicate that post-harvest steps withdraw the positive effects of antibiotic-free rearing on carcass microbiomes. Therefore, it is crucial to assess the contribution of both transport and slaughter on carcass contamination and spreading of ARGs to identify possible mitigation options addressing consumer concerns regarding antimicrobial resistance and enhancing the positive impact of the European legislation, as well as the economic and management efforts of producers to rear antibioticfree chickens.

Supplementary Materials: The following are available online at https:/ /www.mdpi.com/article/10 .3390 / foods11030249/s1, Figure S1: Bray-Curtis dissimilarity plots showing the genera detected on carcasses from the conventional and antibiotic free farm, Figure S2: Bray-Curtis plot beta diversity of functional genes in the carcasses of birds reared in the antibiotic free and conventional farm, Figure S3: Beta diversity of carcasses samples shown as PCoA of Bray-Curtis diversity computed based on the AMR gene family abundances normalized with DESeq2, Table S1: Numbers identifying the tested broilers during slaughtering and corresponding metagenome numbers for the caeca and the carcass samples, Table S2: Normalized mean (DESeq2 normalized counts) of the top 20 genera significantly different $(p<0.05)$ in the caeca of the birds reared in the conventional and antibioticfree farm, Table S3: Mean values of the InvSimpson, Shannon, Chao1 indexes quantified for the genera identified in the caeca of birds from the conventional and the antibiotic free farm, Table S4: Norm_Mean (DESeq2 normalized counts) values of the top 20 functional genes significantly different $(p<0.05)$ in the caeca of the birds reared in the conventional and antibiotic-free farm, Table S5: Norm_Mean values (DESeq2 normalized counts) of the top 20 genera significantly different $(p<0.05)$ in the carcasses of the birds reared in the conventional and antibiotic-free farms, Table S6: Mean values of the InvSimpson, Shannon, Chao1 indexes quantified for the genera identified on carcasses of birds from the conventional and the antibiotic free farm, Table S7: Genes classified as antibiotic resistant genes in the Level 2 category and showing normalized mean values significantly different $(p<0.05)$ in the caeca of birds from the conventional and antibiotic free farm. Genes in bold have been identified also on carcasses but with any statistical significative difference in the two tested groups.

Author Contributions: Conceptualization, A.D.C. and G.M.; methodology, C.O., A.L., F.S. and C.S.; formal analysis C.O., A.L. and C.S.; data curation, C.S.; writing-original draft preparation, A.D.C. and C.O.; writing-review and editing, A.D.C., G.M., F.S. and C.S.; visualization, F.S.; supervision, A.D.C. All authors have read and agreed to the published version of the manuscript.

Funding: This research received no external funding. 
Institutional Review Board Statement: Ethical review and approval were waived for this study due to the fact that organs and carcasses were collected in a commercial slaughterhouse. Live animals were neither reared nor slaughtered for the purpose of the current study.

Data Availability Statement: The 59 metagenomes sequenced in this study are publicly available from MG RAST at https: / www.mg-rast.org/mgmain.html?mgpage=project\&project=mgp 89213 (accessed on 14 December 2021).

Acknowledgments: The authors acknowledge the help received from the workers of the poultry company, their support in the identification of the farms to investigate, in the planning of the slaughtering of the tested groups as first of the day and in the sample collection during evisceration and at the end of the refrigeration tunnel.

Conflicts of Interest: The authors declare that they have no conflict of interest.

\section{References}

1. Eurostat Statistics Explained. Agricultural Production-Livestock and Meat. 2021. Available online: https://ec.europa. eu/eurostat/statistics-explained /index.php?title=Agricultural_production_-_livestock_and_meat\&oldid=427096\#Meat_ production (accessed on 13 October 2021).

2. Marangoni, F.; Corsello, G.; Cricelli, C.; Ferrara, N.; Ghiselli, A.; Lucchin, L.; Poli, A. Role of poultry meat in a balanced diet aimed at maintaining health and wellbeing: An Italian consensus document. Food Nutr. Res. 2015, 59, 27606. [CrossRef] [PubMed]

3. ECDC (European Centre for Disease Prevention and Control); EFSA (European Food Safety Authority); EMA (European Medicines Agency). Third joint inter-agency report on integrated analysis of consumption of antimicrobial agents and occurrence of antimicrobial resistance in bacteria from humans and food-producing animals in the EU/EEA. EFSA J. 2021, 19, 6712. [CrossRef]

4. Brugaletta, G.; De Cesare, A.; Zampiga, M.; Laghi, L.; Oliveri, C.; Zhu, C.; Manfreda, G.; Syed, B.; Valenzuela, L.; Sirri, F. Effects of Alternative Administration Programs of a Synbiotic Supplement on Broiler Performance, Foot Pad Dermatitis, Caecal Microbiota, and Blood Metabolites. Animals 2020, 10, 522. [CrossRef] [PubMed]

5. De Cesare, A.; Caselli, E.; Lucchi, A.; Sala, C.; Parisi, A.; Manfreda, G.; Mazzacane, S. Impact of a probiotic-based cleaning product on the microbiological profile of broiler litters and chicken caeca microbiota. Poult. Sci. J. 2019, 98, 3602-3610. [CrossRef] [PubMed]

6. Durazzi, F.; Sala, C.; Castellani, G.; Manfreda, G.; Remondini, D.; De Cesare, A. Comparison between 16S rRNA and shotgun sequencing data for the taxonomic characterization of the gut microbiota. Sci. Rep. 2021, 11, 3030. [CrossRef]

7. Galloway-Peña, J.; Hanson, B. Tools for analysis of the microbiome. Dig. Dis. Sci. 2020, 65, 674-685. [CrossRef]

8. De Abreu, V.A.; Perdigão, J.; Almeida, S. Metagenomic Approaches to Analyze Antimicrobial Resistance: An Overview. Front. Genet. 2020, 11, 575592. [CrossRef]

9. Wang, Y.; Hu, Y.; Liu, F.; Cao, J.; Lv, N.; Zhu, B.; Zhang, G.; Gao, G.F. Integrated metagenomic and metatranscriptomic profiling reveals differentially expressed resistomes in human, chicken, and pig gut microbiomes. Environ. Int. 2020, 138, 105649. [CrossRef]

10. Roca, I.; Akova, M.; Baquero, F.; Carlet, J.; Cavaleri, M.; Coenen, S.; Cohen, J.; Findlay, D.; Gyssens, I.; Heuer, O.E.; et al. The global threat of antimicrobial resistance: Science for intervention. New Microbes New Infect. 2015, 6, 22-29. [CrossRef]

11. World Health Organization. Antibiotic Resistance. Available online: https://www.who.int/news-room/fact-sheets/detail/ antibiotic-resistance (accessed on 25 September 2020).

12. Cassini, A.; Högberg, L.D.; Plachouras, D.; Quattrocchi, A.; Hoxha, A.; Simonsen, G.S.; Colomb-Cotinat, M.; Kretzschmar, M.E.; Devleesschauwer, B.; Cecchini, M.; et al. Attributable deaths and disability-adjusted life-years caused by infections with antibiotic-resistant bacteria in the EU and the European Economic Area in 2015: A population-level modelling analysis. Lancet Infect. Dis. 2019, 19, 56-66. [CrossRef]

13. Bhogoju, S.; Nahashon, S.; Wang, X.; Darris, C.; Kilonzo-Nthenge, A. A comparative analysis of microbial profile of Guinea fowl and chicken using metagenomic approach. PLoS ONE 2018, 13, e0191029. [CrossRef] [PubMed]

14. Aarestrup, F.M. The livestock reservoir for antimicrobial resistance: A personal view on changing patterns of risks, effects of interventions and the way forward. Philos. Trans. R. Soc. Lond. B Biol. Sci. 2015, 370, 20140085. [CrossRef] [PubMed]

15. Landers, T.F.; Cohen, B.; Wittum, T.E.; Larson, E.L. A review of antibiotic use in food animals: Perspective, policy, and potential. Public Health Rep. 2012, 127, 4-22. [CrossRef] [PubMed]

16. EMA (European Medicines Agency); EFSA (European Food Safety Authority). EMA and EFSA Joint Scientific Opinion on measures to reduce the need to use antimicrobial agents in animal husbandry in the European Union, and the resulting impacts on food safety (RONAFA). [EMA/CVMP/570771/2015]. EFSA J. 2017, 15, e04666. [CrossRef]

17. Tang, K.L.; Caffrey, N.P.; Nóbrega, D.B.; Cork, S.C.; Ronksley, P.E.; Barkema, H.W.; Polachek, A.; Ganshorn, H.; Sharma, N.; Kellner, J.D.; et al. Restricting the use of antibiotics in food-producing animals and its associations with antibiotic resistance in food-producing animals and human beings: A systematic review and meta-analysis. Lancet Planet. Health. 2017, 1, e316-e327. [CrossRef]

18. European Commission. Regulation (EC) No 1831/2003 of the European Parliament and of the Council of 22 September 2003 on additives for use in animal nutrition. Off. J. Eur. Union 2003, 50, 1-15. 
19. European Commission. Regulations 2019/6. Commission Regulation (EU) 2019/6 of the European Parliament and of the Council of 11 December 2018 on veterinary medicinal products and repealing Directive 2001/82/EC (Text with EEA relevance). Off. J. Eur. Union 2019, 6, 1-125.

20. European Commission. Regulation 2019/4. Commission Regulation (EU) 2019/4 of the European Parliament and of the Council of 11 December 2018 on the manufacture, placing on the market and use of medicated feed, amending Regulation (EC) No 183/2005 of the European Parliament and of the Council and repealing Council Directive 90/167/EEC (Text with EEA relevance). Off. J. Eur. Union 2019, 4, 1-23.

21. Xue, H. Efficiently Raising Antibiotic-Free Broilers. Poultry World. 2019. Available online: https://www.poultryworld.net/ Specials / Articles/2019/12/Efficiently-raising-antibiotic-free-broilers513375E/U (accessed on 25 September 2020).

22. De Cesare, A.; Sirri, F.; Manfreda, G.; Moniaci, P.; Giardini, A.; Zampiga, M.; Meluzzi, A. Effect of dietary supplementation with Lactobacillus acidophilus D2/CSL (CECT 4529) on caecum microbioma and productive performance in broiler chickens. PLoS ONE 2017, 12, e0176309. [CrossRef]

23. De Cesare, A.; Parisi, A.; Lucchi, A.; Capozzi, L.; Bianco, A.; Pasquali, F.; Manfreda, G. Microbiota analysis and microbiological hazard assessment in poultry carcasses from conventional and antibiotic free farms. Ital. J. Food Saf. 2019, 7, 7706. [CrossRef]

24. Keegan, K.P.; Glass, E.M.; Meyer, F. MG-RAST, a Metagenomics Service for Analysis of Microbial Community Structure and Function. In Microbial Environmental Genomics (MEG), 1st ed.; Martin, F., Uroz, S., Eds.; Human Press: New York, NY, USA, 2016; Volume 1399, pp. 207-233. [CrossRef]

25. McMurdie, P.J.; Holmes, S. Phyloseq: An R package for reproducible interactive analysis and graphics of microbiome census data. PLOS ONE 2013, 8, e61217. [CrossRef]

26. Love, M.I.; Huber, W.; Anders, S. Moderated estimation of fold change and dispersion for RNA-seq data with DESeq2. Genome Biol. 2014, 15, 550. [CrossRef] [PubMed]

27. Zhu, A.; Ibrahim, J.G.; Love, M.I. Heavy-tailed prior distributions for sequence count data: Removing the noise and preserving large differences. Bioinformatics 2019, 35, 2084-2092. [CrossRef]

28. Benjamini, Y.; Hochberg, Y. Controlling the false discovery rate: A practical and powerful approach to multiple testing. J. R. Stat. Soc. Ser. B Methodol. 1995, 57, 289-300. [CrossRef]

29. Bray, J.R.; Curtis, J.T. An ordination of upland forest communities of southern Wisconsin. Ecol. Monogr. 1957, 27, 325-349. [CrossRef]

30. Alcock, B.P.; Raphenya, A.R.; Lau, T.T.; Tsang, K.K.; Bouchard, M.; Edalatmand, A.; Huynh, W.; Nguyen, A.L.; Cheng, A.A.; Liu, S.; et al. CARD 2020: Antibiotic resistome surveillance with the comprehensive antibiotic resistance database. Nucleic Acids Res. 2020, 48, D517-D525. [CrossRef]

31. Langmead, B.; Salzberg, S.L. Fast gapped-read alignment with Bowtie 2. Nat. Methods 2012, 9, 357-359. [CrossRef]

32. Legendre, P.; Gallagher, E.D. Ecologically meaningful transformations for ordination of species data. Oecologia 2001, 129, 271-280. [CrossRef]

33. Aziz, R.K.; Bartels, D.; Best, A.A.; DeJongh, M.; Disz, T.; Edwards, R.A.; Formsma, K.; Gerdes, S.; Glass, E.M.; Kubal, M.; et al. The RAST Server: Rapid annotations using subsystems technology. BMC Genom. 2008, 9, 75. [CrossRef]

34. Mughini-Gras, L.; Pasqualin, D.; Tarakdjian, J.; Santini, A.; Cunial, G.; Tonellato, F.; Schiavon, E.; Martino, G.D. Short-Term and Long-Term Effects of Antimicrobial Use on Antimicrobial Resistance in Broiler and Turkey Farms. Avian Pathol. 2021, 17, 1-25. [CrossRef]

35. Davies, R.; Wales, A. Antimicrobial resistance on farms: A review including biosecurity and the potential role of disinfectants in resistance selection. Compr. Rev. Food Saf. 2019, 18, 753-774. [CrossRef]

36. EFSA BIOHAZ Panel (EFSA Panel on Biological Hazards); Koutsoumanis, K.; Allende, A.; Alvarez-Ordonez, A.; Bolton, D.; Bover-Cid, S.; Chemaly, M.; Davies, R.; De Cesare, A.; Herman, L.; et al. Scientific Opinion on the role played by the environment in the emergence and spread of antimicrobial resistance (AMR) through the food chain. EFSA J. 2021, 19, 6651. [CrossRef]

37. Althaus, D.; Zweifel, C.; Stephan, R. Analysis of a poultry slaughter process: Influence of process stages on the microbiological contamination of broiler carcasses. Ital. J. Food Saf. 2017, 6, 7097. [CrossRef] [PubMed]

38. Buess, S.; Zurfluh, K.; Stephan, R.; Guldimann, C. Quantitative microbiological slaughter process analysis in a large-scale Swiss poultry abattoir. Food Control 2019, 105, 86-93. [CrossRef]

39. Rasschaert, G.; De Zutter, L.; Herman, L.; Heyndrickx, M. Campylobacter contamination of broilers: The role of transport and slaughterhouse. Int. J. Food Microbiol. 2020, 322, 108564. [CrossRef]

40. Savin, M.; Bierbaum, G.; Hammerl, J.A.; Heinemann, C.; Parcina, M.; Sib, E.; Voigt, A.; Kreyenschmidt, J. Isolation and characterization of ESKAPE-bacteria and ESBL-producing E. coli from waste-and process water of German poultry slaughterhouses. Appl. Environ. Microbiol. 2020, 86, e02748-19. [CrossRef]

41. Li, S.; Mann, D.A.; Zhang, S.; Qi, Y.; Meinersmann, R.J.; Deng, X. Microbiome-Informed Food Safety and Quality: Longitudinal Consistency and Cross-Sectional Distinctiveness of Retail Chicken Breast Microbiomes. Msystems 2020, 5, e00589-20. [CrossRef]

42. Anadón, A.; Martinez-Larrañaga, M.R.; Diaz, M.J.; Bringas, P.; Fernandez, M.C.; Martinez, M.A.; Fernandez-Cruz, M.L. Pharmacokinetics of amoxicillin in broiler chickens. Avian Pathol. 1996, 25, 449-458. [CrossRef]

43. Spielmeyer, A.; Ahlborn, J.; Hamscher, G. Simultaneous determination of 14 sulfonamides and tetracyclines in biogas plants by liquid-liquid-extraction and liquid chromatography tandem mass spectrometry. Anal. Bioanal. Chem. 2014, 406, 2513-2524. [CrossRef] 
44. Feye, K.M.; Baxter, M.F.A.; Tellez-Isaias, G.; Kogut, M.H.; Ricke, S.C. Influential factors on the composition of the conventionally raised broiler gastrointestinal microbiomes. Poult. Sci. 2020, 99, 653-659. [CrossRef]

45. Fernández-Rubio, C.; Ordóñez, C.; Abad-González, J.; Garcia-Gallego, A.; Honrubia, M.P.; Mallo, J.J.; Balaña-Fouce, R. Butyric acid-based feed additives help protect broiler chickens from Salmonella Enteritidis infection. Poult. Sci. 2009, 88, 943-948. [CrossRef]

46. Dalmasso, G.; Nguyen, H.T.; Yan, Y.; Charrier-Hisamuddin, L.; Sitaraman, S.V.; Merlin, D. Butyrate transcriptionally enhances peptide transporter PepT1 expression and activity. PLoS ONE 2008, 3, e2476. [CrossRef]

47. Blanco, P.; Hernando-Amado, S.; Reales-Calderon, J.A.; Corona, F.; Lira, F.; Alcalde-Rico, M.; Bernardini, A.; Sanchez, M.B.; Martinez, J.L. Bacterial multidrug efflux pumps: Much more than antibiotic resistance determinants. Microorganisms 2016, 4, 14. [CrossRef]

48. Marquez, B. Bacterial efflux systems and efflux pumps inhibitors. Biochimie 2005, 87, 1137-1147. [CrossRef]

49. Seeger, M.A.; Schiefner, A.; Eicher, T.; Verrey, F.; Diederichs, K.; Pos, K.M. Structural asymmetry of AcrB trimer suggests a peristaltic pump mechanism. Science 2006, 313, 1295-1298. [CrossRef] [PubMed]

50. Munk, P.; Knudsen, B.E.; Lukjancenko, O.; Duarte, A.S.R.; Van Gompel, L.; Luiken, R.E.C.; Smit, L.A.M.; Schmitt, H.; Dorado Garcia, A.; Borup Hansen, R.; et al. Abundance and diversity of the fecal resistome in slaughter pigs and broilers in nine European countries. Nat. Microbiol. 2018, 3, 898-908. [CrossRef] [PubMed]

51. EFSA BIOHAZ Panel (EFSA Panel on Biological Hazards). Scientific Opinion on the evaluation of the safety and efficacy of peroxyacetic acid solutions for reduction of pathogens on poultry carcasses and meat. EFSA J. 2014, 12, 3599. [CrossRef]

52. Di Francesco, C.E.; Smoglica, C.; Profeta, F.; Farooq, M.; Di Giannatale, E.; Toscani, T.; Marsilio, F. Research Note: Detection of Antibiotic-Resistance Genes in Commercial Poultry and Turkey Flocks from Italy. Poult. Sci. 2021, 100, 101084. [CrossRef] [PubMed]

53. EFSA and ECDC (European Food Safety Authority and European Centre for Disease Prevention and Control). The European Union Summary Report on Antimicrobial Resistance in zoonotic and indicator bacteria from humans, animals and food in 2018/2019. EFSA J. 2021, 19, 6490. [CrossRef] 